\title{
Rectal stenosis caused by cytomegalovirus colitis
}

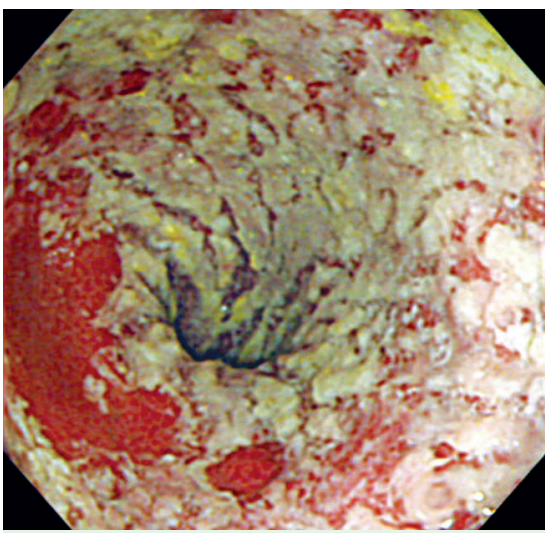

Fig. 1 Colonoscopy showing multiple superficial ulcerations in the rectum, $3-6 \mathrm{~mm}$ in size.

A 87-year-old man attended our hospital for watery diarrhea since 1 week. Colonoscopy revealed multiple $3-6 \mathrm{~mm}$ superficial ulcerations in the rectum ( $\bullet$ Fig. 1). The ulcers were shallow and had sharp margins (†ig. 2). Cytomegalovirus (CMV) pp65 antigenemia assay (C7-HRP; SRL Inc, Japan) revealed many CMV-positive cells (6/1400 cells). Because CMV viremia was strongly suspected, treatment was initiated with ganciclovir. Immunohistochemistry of biopsy specimens taken from the ulcer craters revealed intranuclear inclusions that were positive for immunostaining with specific antiCMV antibodies. After 7 days, the diarrhea ceased. At follow-up 2 months later, colonoscopy ( Fig. 3) and barium enema ( $\bullet$ Fig. 4) both revealed pinpoint stenosis at the rectosigmoid junction, related to the ulcer scars. We took this to mean a cure in endoscopic terms.

$\mathrm{CMV}$ is an important and ubiquitous herpes virus, and the gastrointestinal tract is one of the common sites of infection [1]. Colonoscopy in our case revealed superficial ulcerative colitis. The pathogenesis of CMV-induced ulcerations is thought to involve ischemic mucosal injury secondary to infection of vascular endothelial cells [2]. In addition, it is well known that rectal stenosis is caused by inflammatory bowel disease such as ulcerative colitis and Crohn's disease [3,4]. However, in our case, we thought that the stenosis was a result of the circumferential location of the rectal ulcers. To our knowledge, this is the first reported case of rectal stenosis caused by CMV colitis.

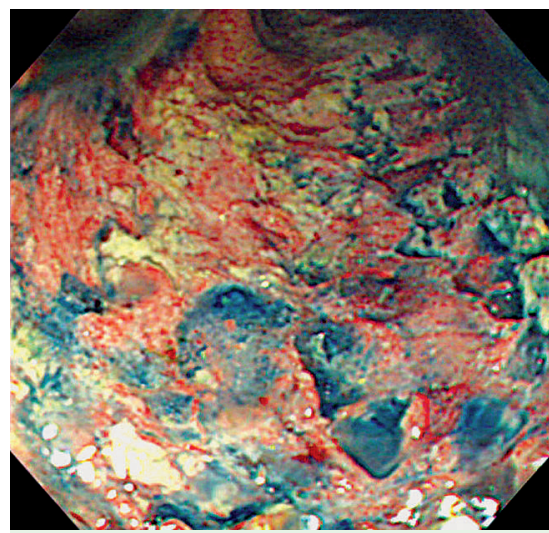

Fig. 2 Colonoscopy with indigo carmine stain revealed shallow ulcerations with sharp margins in the rectum.

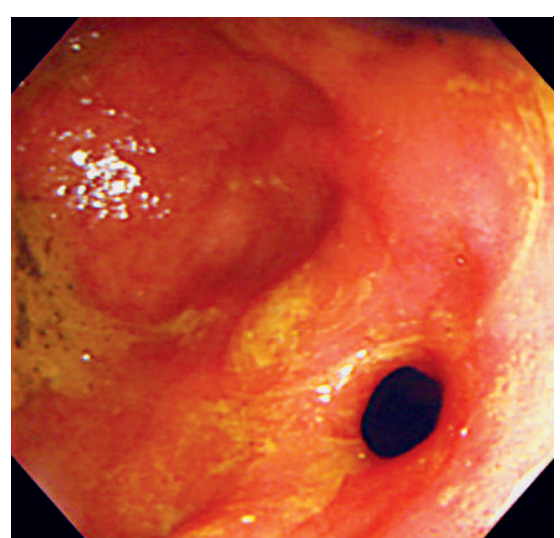

Fig. 3 Colonoscopy after 2 months showing pinpoint stenosis as a result of scarring of ulcers at the rectosigmoid junction.

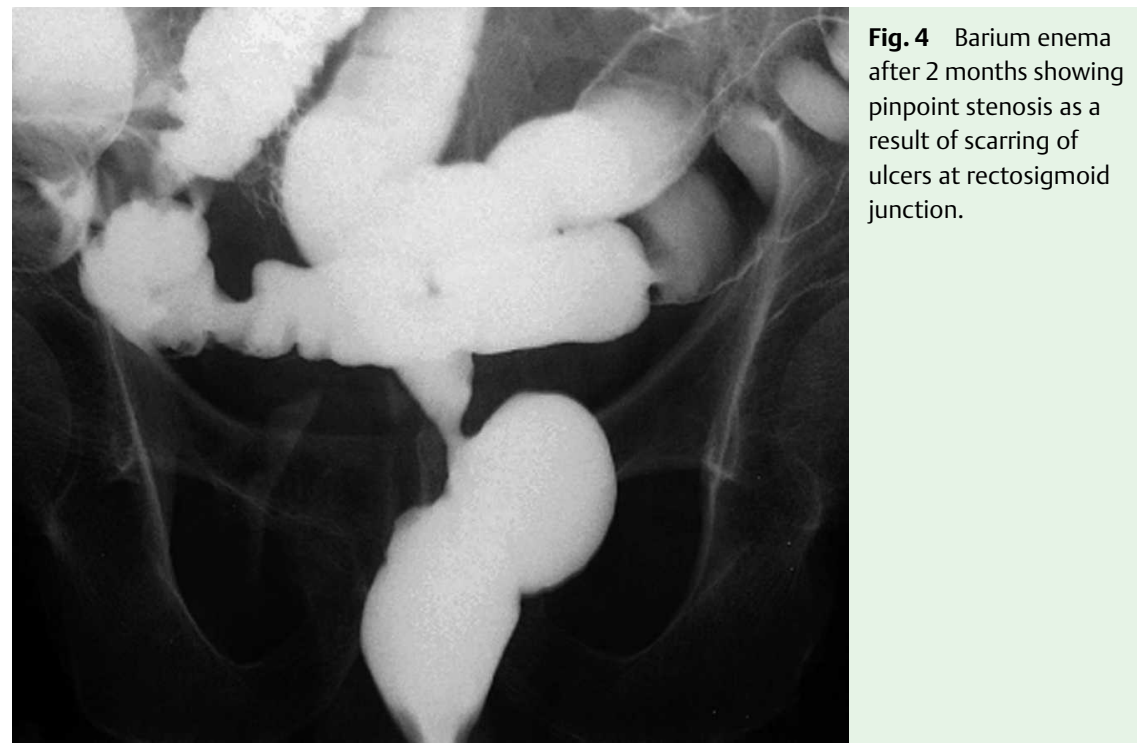

Endoscopy_UCTN_Code_CCL_1AD_2AZ

\section{K. Kume, I. Yoshikawa}

Third Department of Internal Medicine, University of Occupational and Environmental Health, School of Medicine, Kitakyusyu, Japan

\section{Reference}

1 Teixidor HT, Honing CL, Norsoph E et al. Cytomegalovirus infection of the alimentary canal: radiologic findings with pathologic correlation. Radiology 1987; 163: $317-323$

2 Cheung AN, Ng IO. Cytomegalovirus infection of the gastrointestinal tract in nonAIDS patients. Am J Gastroenterol 1993; 88: $1882-1886$

3 Grobler SP, Hosie KB, Affie E et al. Outcome of restorative proctocolectomy when the diagnosis is suggestive of Crohn's disease. Gut 1993; 34: $1384-1388$
4 Gumaste V, Sachar DB, Greenstein AJ. Benign and malignant colorectal strictures in ulcerative colitis. Gut 1992; 33: 938-941

Bibliography

DOI $10.1055 / \mathrm{s}-0029-1214801$

Endoscopy 2009; 41: E197

(c) Georg Thieme Verlag KG Stuttgart · New York ISSN 0013-726X

\section{Corresponding author}

\section{K. Kume, MD, PhD}

Third Department of Internal Medicine University of Occupational and Environmental Health, School of Medicine

1-1 Iseigaoka

Yahatanishi-ku

Kitakyusyu 807-8555

Japan

Fax: +81-93-692-0107

k-kume@med.uoeh-u.ac.jp 